\title{
EQ-5D-5L Health-State Values for the Mexican Population
}

\author{
Cristina Gutierrez-Delgado, ${ }^{1,2}$ Rosa-María Galindo-Suárez ${ }^{3,4} \cdot$ Cesar Cruz-Santiago $^{3,4} \cdot$ Koonal Shah $^{5} \cdot$ \\ Manny Papadimitropoulos ${ }^{6,7} \cdot$ Yan Feng $^{8} \cdot$ Bernarda Zamora $^{9} \cdot$ Nancy Devlin $^{9,10}$
}

Accepted: 14 March 2021 / Published online: 26 June 2021

(c) The Author(s) 2021

\begin{abstract}
Objective To generate a value set for the Mexican adult general population to support and facilitate the inclusion of qualityadjusted life years (QALYs) into the health technology assessment process of the Mexican healthcare authorities.

Methods A representative sample of the Mexican adult population stratified by age, sex and socio-economic status was used. Following version 2.0 of the EuroQol EQ-5D-5L valuation protocol, trained interviewers guided participants in completing composite time trade-off (cTTO) and discrete-choice experiment (DCE) tasks included in the EQ-VT software. Generalized least squares, Tobit and Bayesian models were used for cTTO data. The choice of value set model was based on criteria that included: theoretical considerations, parsimony, logical ordering of coefficients, and statistical significance.

Results Based on quality control criteria and interviewer judgment, 1000 out of 1032 participants provided useable responses. Participants' demographic characteristics were similar to the 2010 Mexican Population Census and followed the socioeconomic structure defined by the Mexican Association of Marketing Research and Public Opinion Agencies (AMAI). The predicted index values in the final cTTO model (a heteroscedastic censored model with Bayesian estimation) ranged from -0.5960 to 1 , with $19.7 \%$ of all predicted health state scores less than 0 (i.e., worse than dead).

Conclusion This study has generated the first value set representing the stated preferences of the Mexican adult population for use in estimating QALYs. The resulting EQ-5D-5L value set is technically robust and will facilitate health economic analyses as well as quality-of-life studies.
\end{abstract}

\section{Key Points for Decision Makers}

The EQ-5D-5L Mexican value set will facilitate both cross-country comparisons and inclusion of Mexican participants in international multicenter clinical trials of new medical technologies and quality-of-life studies.

The Mexican value set will facilitate the inclusion of QALYs into the HTA process that Mexican healthcare authorities apply for new medical technologies to be financed by public healthcare institutions.

The Mexican value set will allow use of EQ-5D-5L, a preference-based health-related quality-of-life measure, to quantify patients' health outcomes in Mexico. These data can be used in health economic analysis and contribute to the monitoring of healthcare service quality in the Mexican context.

Cristina Gutierrez-Delgado

crisgutdel@gmail.com

Extended author information available on the last page of the article

\section{Introduction}

Mexico, with a population of just over 125 million in 2018 [1], is considered the second biggest market for medical technology in Latin America, just behind Brazil [2]. Since 2003, the General Health Council (GHC) has positioned itself as the main health technology assessor (decision maker) of the public institutions given that it is this collegiate body's sole responsibility to constantly maintain and update the Basic Formulary of Medications and Healthcare Supplies Catalogue (BFMHSC) [3-7]. This document groups, characterizes, and encodes the drugs, medical materials, instruments, medical equipment, and diagnostics used by the National Health System's public institutions to provide health services to the population. In 2020 the GHC decided to transform the BFMHSC into the National Compendium of Healthcare Supplies ("Compendium"). The Compendium aims to strengthen the evaluation of healthcare, to optimize public resources directed at addressing health problems in the country, and to notify and update health professionals. The GHC periodically updates 
the health technology assessment (HTA) processes used to determine inclusion in the Compendium.

HTA in Mexico has relied mainly on cost per life-year analyses. However, the GHC wishes to extend this to include quality of life and quality-adjusted life years (QALYs). To include evidence on cost per QALYs gained, it is necessary to measure and value health-related quality of life. Several generic preference-based measures of health-related quality of life exist for this purpose. One of the most widely used, internationally, for the purpose of facilitating cost-effectiveness analysis, is the EQ-5D-5L [8]. The EQ-5D-5L summarizes health in terms of five dimensions (mobility, self-care, usual activities, pain/discomfort, anxiety/depression) and five levels of problems (no $=1$, slight $=2$, moderate $=3$, severe $=4$, extreme/unable to $=5$ ). Its use in HTA involves collection of EQ-5D-5L data from patients (e.g., in clinical trials) and summarizing those data using a "value set." The value sets indicate how good or bad each health state is, on a scale anchored at 1 (full health) and 0 (dead), as required for the estimation of QALYs. The value sets are generally based on the views of a general public sample, obtained using stated preference elicitation methods [9].

The GHC is strongly interested in adopting results from an internationally well-established methodology for valuing health-related quality of life, suitable for routine use in HTA. Hence, the GHC supported a project developed by the Economic Analysis Unit (EAU) of the Mexican Ministry of Health to estimate the first EQ-5D-5L value set for the Mexican population using the EuroQol Group's international EQ-5D-5L valuation protocol and software. Rationales for GHC and EAU selecting the EQ-5D-5L include the widespread use of the instrument worldwide in cost-utility analysis [10], and its acceptance by key HTA bodies for use in evidence submitted to their decision-making processes [11-13]. The EQ-5D-5L is also suitable for use in population health studies, and in routine outcomes measurement in health-care systems (such as the English NHS PROMs programme and Swedish National Quality Registers) [14, 15]. A further rationale for selecting the EQ-5D-5L is the availability of an international protocol to support and guide the generation of a value set. This protocol has been used to generate EQ-5D-5L value sets in over 20 countries to date, and its methods and processes for quality control have been refined and strengthened to ensure that population preferences are captured in a robust manner, which is required for application in HTA [16].

To address the GHC's requirements, the main objective of this study was to generate a value set for the Mexican adult general population to support and facilitate the inclusion of QALYs into the HTA process of the Mexican healthcare authorities.

\section{Methods}

\subsection{Study Design}

A nationally representative sampling of the Mexican adults (18 years and older) stratified by sex, age, and socioeconomic level was designed and used. The design followed geographical and population frames developed by CONAPO [1], the Mexican Office of Statistics and Geography [17], as well as the socioeconomic classification of households of the Mexican Association of Marketing Research and Public Opinion Agencies (AMAI) [18]. To ensure the sample size of 1,000 completed interviews recommended by the EQ$5 \mathrm{D}-5 \mathrm{~L}$ valuation protocol, a $15 \%$ non-response rate was considered (see detailed sampling design in Section 1 of the ESM Appendix).

Two methods were used to elicit stated preferences from the sample: (a) composite time trade-off (cTTO), comprising "conventional" TTO to obtain values $\geq 0$, and a "leadtime TTO" to obtain values $<0$ [19], and (b) discrete-choice experiments, involving pairwise choices between health states. Face-to-face computer-assisted interviews were undertaken with the Mexican general public aged 18 years and over. The study design, sampling, and data quality monitoring followed the EQ-5D-5L valuation protocol version 2.0 that has emerged as best practice from previous studies [14]. This included a training process for the Mexican study team and weekly follow-up meetings with EuroQol Office scientists during the data collection phase. It also recommends a minimum sample of 1000 useable respondents [20].

A prior decision was made to base the Mexican value set on cTTO data only, as long as the cTTO data were of high quality and the resulting value set showed desirable characteristics such as logically ordered parameter estimates. This is in line with the approach used in many other countries' value-set studies (e.g., [21-25]). DCE data alone can be used to obtain values on a latent scale, but these do not meet the conventions of QALY estimation [26]. cTTO and DCE data can be combined via hybrid modelling [27], but there is a lack of consensus about the merits of this approach, and, notably, the new UK value set for EQ-5D-5L now underway is based on cTTO only [28]. The design, methods, results, and analyses relating to the DCE data are reported in Section 2 of the ESM Appendix.

\subsection{Valuation Intervention and Methods of Eliciting Preferences}

The EuroQol Valuation Technology (EQ-VT) software captured respondents' preferences regarding EQ-5D-5L health states. A sub-set of 86 health states were valued directly in the cTTO tasks. From this sub-set, blocks of ten health 
states were generated, each containing the worst health state (55555), one mild state (level 2 in one dimension only), and eight states that varied in severity. Further details about the experimental design are reported by Oppe and van Hout [20]. The software randomly assigned participants to cTTO blocks.

\subsection{Data Collection and Quality Control Process}

Data were collected in the period June-August 2019 by a team of 15 interviewers. The interviewers were chosen from 42 candidates who had been recruited by a public opinion agency (De las Heras-Demotecnia). Selection criteria included possession of a social science background (sociologists, social anthropologist, social workers, psychologists, historians) and experience in in-depth interviewing. During the training process two interviewers opted out for personal reasons. Another was discharged during the pilot phase for not meeting the required quality standards [29].

\subsection{Data Exclusions}

Prior to commencing data analysis, the study team agreed to exclude two sets of data on quality grounds: (a) cTTO data for any respondents who gave the same value for all ten health states; and (b) any cTTO observations flagged for exclusion by the respondent via the feedback module (a feature in the EQ-VT that allows respondents to view the rank ordering of health states that would be inferred from their cTTO responses, and offers them the opportunity to flag any health states they feel, on reflection, may have been evaluate inappropriately). The former exclusion rule is commonly used in value set studies [30]; the latter has regularly been used in recent EQ-5D-5L valuation studies following the introduction of the feedback module (e.g., [31]).

Interviewer effects were assessed, for example, by comparing interviewers' cTTO value distributions. If the data for a given interviewer showed highly unusual patterns or suggested protocol non-compliance, then those data were excluded on quality grounds. However, such effects were assessed throughout the data collection phase. This included analysing the interim data on a weekly basis and providing regular feedback to interviewers about their performance (such as whether they were spending sufficient time explaining the TTO task to respondents), in line with best practice [29], thereby minimising the need for data exclusions.

\subsection{Data Analysis and Modelling}

Descriptive analyses were used to examine responses to the tasks. For cTTO, this included inspecting the overall distribution of values and calculating means, medians, and standard deviations, both for each health state and grouped by level sum score (LSS; sum of the five dimension levelse.g., health state 13122 has an LSS of $1+3+1+2+2=$ $9)$. The proportion of respondents with logical inconsistencies in their cTTO data-where a higher value was given to A than to B when B is at least as good as A across all dimensions-was also examined.

The data modelling strategy sought to take three key features of the cTTO data into account. First, the minimum cTTO value is bounded at -1 by design but respondents might have traded more time in full health had they been given the opportunity to do so, so responses were treated as left-censored at -1 . Second, each respondent undertook ten cTTO tasks so the modelling accounted for the possibility that observations from the same respondent would be more similar than those from other respondents. Third, it is commonly observed that the variance of TTO values increases as the severity of the health states increases (see [26, 31, 32]). The extent to which this was the case in the Mexican data was examined by calculating the standard deviations of residuals (from a 20-parameter generalized least-squares (GLS) model) by health state, and modelling sought to account for this heteroscedasticity.

Four 20-parameter main effects cTTO models were estimated. Model 1 was estimated by a GLS regression. The model accounted for the second feature of the cTTO data noted above, i.e., observations from the same respondent might be more similar than those from other respondents. Model 2 was estimated by a Tobit regression. The model addressed features 1 (i.e., cTTO data were censored at -1 ) and 2 of the data. Model 3 is a heteroscedastic model with Bayesian estimation that accounted for features 2 and 3 (i.e., the variance of TTO values might increase as the severity of the health states increase) of the cTTO data. Model 4 addressed all three features of the cTTO data using a heteroscedastic censored model with Bayesian estimation. The estimation was conducted using the Markov chain Monte Carlo simulation (MCMC) method with random walk Metropolis-Hastings algorithm [33]. To confirm the presence of feature 3 in the cTTO data, we checked whether the variance of residuals in the 20-parameter main effects GLS model was constant. Final model selection was informed by theoretical considerations relating to the characteristics of the cTTO data, logical ordering of the parameter estimates (i.e., larger decrements are expected for worse problems), significance of the parameters, and relevant information criteria (i.e., Bayesian information criterion (BIC), Akaike information criterion (AIC), Deviance information criterion (DIC)). As model 4 addressed all features of the cTTO data, we present its specification in detail below:

The latent variable $\mathrm{cTTO}_{i j}^{*}$ is censored at -1 . As a result, the observed variable $\mathrm{cTTO}_{i j}$ can only have values no less than the censored value. 
$\mathrm{cTTO}_{i j}=\left\{\begin{array}{cl}\mathrm{cTTO}_{i j}^{*} & \text { if } \mathrm{cTTO}_{i j}^{*}>-1 \\ -1 & \text { if } \mathrm{cTTO}_{i j}^{*} \leq-1\end{array}\right.$.

A 20-parameter model was estimated in modelling the observed $c T T O_{i j}$ using Eq. (1).

$$
\begin{aligned}
\mathrm{cTTO}_{i j}= & 1-\left(\beta_{1} \mathrm{MO}_{j}+\beta_{2} \mathrm{MO}_{j}+\beta_{3} \mathrm{MO}_{j}+\beta_{4} \mathrm{MO}_{j}\right. \\
& +\beta_{5} \mathrm{SC}_{j}+\beta_{6} \mathrm{SC}_{j}+\beta_{7} \mathrm{SC}_{j}+\beta_{8} \mathrm{SC}_{j} \\
& +\beta_{9} \mathrm{UA}_{j}+\beta_{10} \mathrm{UA} 3_{j}+\beta_{11} \mathrm{UA}_{j}+\beta_{12} \mathrm{UA}_{j} \\
& +\beta_{13} \mathrm{PD}_{j}+\beta_{14} \mathrm{PD}_{j}+\beta_{15} \mathrm{PD}_{j}+\beta_{16} \mathrm{PD}_{j} \\
& \left.+\beta_{17} \mathrm{AD} 2_{j}+\beta_{18} \mathrm{AD} 3_{j}+\beta_{19} \mathrm{AD} 4_{j}+\beta_{20} \mathrm{AD} 5_{j}\right)+u_{i}+\varepsilon_{i j}
\end{aligned}
$$

$u_{i} \sim \operatorname{iid} N\left(0, \sigma_{u}^{2}\right)$

$\varepsilon_{i j} \sim \operatorname{iid} N\left(0, \sigma_{j}^{2}\right)$

where the 20 parameters for $\beta$ reflect the decrement in utility from full health as problems reported in each level and dimension of a health state. The utility for a health state is described by 20 dummies, i.e., MO2-MO5 (Mobility levels 2-5), SC2-SC5 (Self-Care levels 2-5), UA2-UA5 (Usual Activities levels 2-5), PD2-PD5 (Pain/Discomfort levels 2-5), and AD2-AD5 (Anxiety and Depression levels 2-5), with value equal to one if the category applies to the description of the health state and zero otherwise; $u_{i}$ is the respondent level random intercept, $\varepsilon_{i j}$ is a heteroscedastic error term, subscript $i$ refers to a respondent, subscript $j$ accounts for each valuation task completed, iid refers to independent and identically distributed.

The heteroscedastic error term is specified by Eq. (2), which allows for an exponential relationship between the variance of the cTTO values and the severity of health states.

$$
\begin{aligned}
\sigma_{j}^{2}= & \exp \left(\alpha_{0}+\alpha_{1}\left(1-\left(\beta_{1} \mathrm{MO}_{j}+\beta_{2} \mathrm{MO}_{j}+\beta_{3} \mathrm{MO}_{j}+\beta_{4} \mathrm{MO}_{j}\right.\right.\right. \\
& +\beta_{5} \mathrm{SC}_{j}+\beta_{6} \mathrm{SC}_{j}+\beta_{7} \mathrm{SC}_{j}+\beta_{8} \mathrm{SC}_{j} \\
& +\beta_{9} \mathrm{UA} 2_{j}+\beta_{10} \mathrm{UA} 3_{j}+\beta_{11} \mathrm{UA}_{j}+\beta_{12} \mathrm{UA}_{j} \\
& +\beta_{13} \mathrm{PD}_{j}+\beta_{14} \mathrm{PD}_{j}+\beta_{15} \mathrm{PD}_{j}+\beta_{16} \mathrm{PD}_{j} \\
& \left.\left.\left.+\beta_{17} \mathrm{AD} 2_{j}+\beta_{18} \mathrm{AD} 3_{j}+\beta_{19} \mathrm{AD}_{j}+\beta_{20} \mathrm{AD} 5_{j}\right)\right)\right)
\end{aligned}
$$

All models were run with and without applying the exclusion criteria. Comparisons were made with the value sets of other selected countries.

STATA/MP 16.0 was used for all statistical analysis.

\section{Results}

The mean interview duration-including background questions and task explanations as well as the cTTO and DCE tasks themselves-was 44.2 min (SD: 19.0 min; median: $41.9 \mathrm{~min}$ ). Of the sample, $38.0 \%$ self-reported being in health state 11111 . Table 1 summarizes the background characteristics of the sample.

\subsection{Data Characteristics}

Using the feedback module, $2.1 \%$ of cTTO responses were flagged as problematic. These responses were excluded, leaving 9787 cTTO observations in the dataset for analysis. No other data were excluded. No respondent gave the same value for all health states. The majority of respondents $(70.6 \%)$ had no logical inconsistencies within their cTTO data. Before excluding responses flagged via the feedback module, $67.3 \%$ of respondents had no logical inconsistencies.

The cTTO values and standard deviations increased with LSS. The mean observed cTTO values ranged from 0.96 (for 11121) to -0.66 (for 55555). Four health states out of the 86 that were valued directly $-55555,52455,44553$, and 43555 - had a mean value of $<0$. Of the 9787 cTTO valuations, $1925(19.7 \%)$ were $<0$. The proportions of values clustered at $-1,0$, and 1 were $6.9 \%, 1.3 \%$, and $4.5 \%$, respectively. Descriptive statistics of the values, by health state and by LSS, as well as the value distribution, are shown in Tables 1 and 2 of Section 3 in the ESM Appendix. The histogram of observed cTTO values is presented in Fig. 1 of Section 3 in the ESM Appendix.

\subsection{Modelling Results}

We checked whether our cTTO data show increased variance as the severity of the health states increases. This data feature was validated in two ways. First, in the test of whether the variance of residuals in the 20-parameter main effects GLS model was constant, the null hypothesis was rejected [ $p$ $\left.<0.0001 ; \chi^{2}(1000)=32429.5\right]$. Second, it was shown graphically that the standard deviation of residuals was greater for more severe health states (see Fig. 3 of Section 3 in the ESM Appendix). As the cTTO data presented all three features noted above, the chosen model should be able to address them all. Our preferred model is model 4, i.e., the Bayesian heteroscedasticity model with censoring at -1 .

Table 2 shows the cTTO results using the preferred model. Just under $4.5 \%$ of all TTO observations were leftcensored. Excluding observations flagged using the feedback module lowered the deviance information criterion (DIC) but did not substantially affect the model results- the only 
Table 1 Sample background characteristics

\begin{tabular}{|c|c|c|}
\hline & All respondents $(n=1000)$ & $\begin{array}{l}\text { General popu- } \\
\text { lation }(N= \\
78,423,336)\end{array}$ \\
\hline & $N(\%)$ & $N(\%)$ \\
\hline \multicolumn{3}{|l|}{ Age, years } \\
\hline $18-24$ & $223(22.3)$ & $15,620,430(19.9)$ \\
\hline $25-34$ & $261(26.1)$ & $18,849,051(24.0)$ \\
\hline $35-44$ & $198(19.8)$ & $16,712,012(21.3)$ \\
\hline $45-54$ & $130(13.0)$ & $12,005812(15.3)$ \\
\hline $55-64$ & $100(10.0)$ & $7,657,834(9.8)$ \\
\hline $65-74$ & $59(5.9)$ & $4,577,336(5.8)$ \\
\hline 75 and above & $29(2.9)$ & $3,000,862(3.8)$ \\
\hline \multicolumn{3}{|l|}{ Gender } \\
\hline Male & $486(48.6)$ & $37,704,417(48.1)$ \\
\hline Female & $514(51.4)$ & $40,718,919$ (51.9) \\
\hline \multicolumn{3}{|l|}{ Region } \\
\hline Region 1 & $100(10.0)$ & $7,931,675(10.1)$ \\
\hline Region 2 & $192(19.2)$ & $15,093,816(19.2)$ \\
\hline Region 3 & $126(12.6)$ & $9,682,184(12.3)$ \\
\hline Region 4 & $237(23.7)$ & $18,697,285(23.8)$ \\
\hline Region 5 & $118(11.8)$ & $9,124,117(11.6)$ \\
\hline Region 6 & $145(14.5)$ & $11,178,743(14.3)$ \\
\hline Region 7 & $82(8.2)$ & $6,715,516(8.6)$ \\
\hline \multicolumn{3}{|c|}{ Socioeconomic group } \\
\hline $\mathrm{A} / \mathrm{B}$ & $107(10.7)$ & $7,920,757(10.1)$ \\
\hline $\mathrm{C}+$ & 297 (29.7) & $22,664,344(28.9)$ \\
\hline $\mathrm{C}$ & $151(15.1)$ & $12,312,464(15.7)$ \\
\hline $\mathrm{C}-$ & $142(14.2)$ & $11,292,960(14.4)$ \\
\hline $\mathrm{D}+$ & $131(13.1)$ & $10,587,150(13.5)$ \\
\hline $\mathrm{D}$ & $111(11.1)$ & $8,783,414(11.2)$ \\
\hline E & $61(6.1)$ & $4,862,247(6.2)$ \\
\hline \multicolumn{3}{|c|}{ Self-rated mobility } \\
\hline Level 1 & $770(77.0)$ & NA \\
\hline Level 2 & $148(14.8)$ & \\
\hline Level 3 & $59(5.9)$ & \\
\hline Level 4 & $16(1.6)$ & \\
\hline Level 5 & $7(0.7)$ & \\
\hline \multicolumn{3}{|c|}{ Self-rated self-care } \\
\hline Level 1 & $935(93.50)$ & \\
\hline Level 2 & $43(4.30)$ & NA \\
\hline Level 3 & $13(1.30)$ & \\
\hline Level 4 & $6(0.60)$ & \\
\hline Level 5 & $3(0.30)$ & \\
\hline \multicolumn{3}{|c|}{ Self-rated usual activities } \\
\hline Level 1 & $818(81.80)$ & \\
\hline Level 2 & $123(12.30)$ & NA \\
\hline Level 3 & $43(4.30)$ & \\
\hline Level 4 & $13(1.30)$ & \\
\hline Level 5 & $3(0.30)$ & \\
\hline \multicolumn{3}{|c|}{ Self-rated pain/discomfort } \\
\hline Level 1 & $555(55.50)$ & \\
\hline Level 2 & $299(29.90)$ & NA \\
\hline
\end{tabular}


Table 1 (continued)

\begin{tabular}{lll}
\hline & All respondents $(n=1000)$ & $\begin{array}{c}\text { General popu- } \\
\text { lation }(N= \\
78,423,336)\end{array}$ \\
& $N(\%)$ & $N(\%)$ \\
\hline Level 3 & $107(10.70)$ & \\
Level 4 & $36(3.60)$ & \\
Level 5 & $3(0.30)$ & \\
Self-rated anxiety/depression & & \\
Level 1 & $661(66.10)$ & \\
Level 2 & $242(24.20)$ & \\
Level 3 & $77(7.70)$ & \\
Level 4 & $19(1.90)$ & NA \\
Level 5 & $1(0.10)$ & NA \\
Self-rated EQ-5D-5L health state & & \\
Health state 11111 & $380(38.00)$ & \\
Any other health state & $620(62.00)$ & \\
Self-rated EQ-VAS rating & Mean $=83.2 ;$ Median = 90.0; SD = & \\
& 15.5 & \\
\hline
\end{tabular}

Population projections based upon the Mexican Population Census 2010. For further details on Region and Socioeconomic group please refer to the ESM Appendix

minor change was that without exclusions Anxiety/Depression 2 (AD2) had a marginally smaller coefficient than Pain/ Discomfort 2 (PD2), whereas after exclusions the AD2 coefficient was marginally larger. All of the coefficients are logically ordered - that is, for any given dimension a higher level of problems has a larger coefficient (and therefore confers more disutility) than a smaller level.

The MCMC sample size is 10,000. It is the result of $12,500 \mathrm{MCMC}$ iterations in total with the first 2500 iterations discarded. The diagnostics of MCMC suggests good performance of the preferred model. The acceptance rate is reported as $43.91 \%$. The average efficiency is $7.9 \%$.

In the other models estimated (see Table 3 in Section 3 of the ESM Appendix), the coefficient for Mobility 2 (MO2) is negative but non-significant; all other coefficients are logically ordered and consistent with those of the preferred model. In all models, the largest utility decrement for a dimension-level is for PD5; the smallest is for MO2. There are large utility decrements in the moves from PD4 and AD4 to PD5 and AD5, respectively.

\subsection{Using the Preferred Value-Set Model to Calculate EQ-5D-5L Health-State Values}

The EQ-5D-5L Mexican value set is based on cTTO data (with exclusions applied) modelled using the Bayesian heteroscedasticity model with censoring at -1 (Table 2). To apply this model as an algorithm for obtaining EQ-5D-5L health-state values, the parameter estimates for each relevant dimension-level combination should be subtracted from 1 (which represents full health). For example, the value for health state 12345 is $1-0-0.0476-0.0952-0.2283-0.3337$ $=0.2952$.

The value set ranges from 0.984 for 21111 (mildest health state other than full health, describing slight problems in walking about and no problems on the other dimensions) to -0.596 for 55555 (worst health state in the descriptive system). Based on the sizes of level 5 coefficients, the most important dimension is pain/discomfort, followed by anxiety/depression, usual activities, mobility, and self-care.

\subsection{Comparison with Value Sets of Other Countries}

The results from the Mexican study can be compared to those of Uruguay [21], the first Latin American country to undertake an EQ-5D-5L valuation study; and the USA [25], Mexico's neighbour to the north (Table 3). All three countries have based their value sets on cTTO data only. Mexico sits halfway between Uruguay and the USA in terms of the proportion of EQ-5D-5L health states with a modelled value of less than zero, though its minimum value of -0.596 is the lowest of the three countries. Compared to the value sets of Uruguay and the USA, the Mexican value set places greater importance on anxiety/depression and usual activities, and less importance on mobility and self-care.

Another Latin American country-Peru-recently completed an EQ-5D-5L value-set study, albeit using a "Lite" protocol that is less reliant on the cTTO [34]. 
Table 2 cTTO model results: Heteroscedastic censored model with Bayesian estimation

\begin{tabular}{|c|c|c|c|c|}
\hline \multirow[t]{2}{*}{ Dimension-level } & \multicolumn{2}{|c|}{ No exclusions } & \multicolumn{2}{|c|}{$\begin{array}{l}\text { With exclusion criteria } \\
\text { applied }\end{array}$} \\
\hline & Coefficients & MCSE & Coefficients & MCSE \\
\hline Mobility 2 & 0.0168 & 0.000310 & 0.0160 & 0.000297 \\
\hline Mobility 3 & 0.0458 & 0.000427 & 0.0473 & 0.000403 \\
\hline Mobility 4 & 0.1727 & 0.000461 & 0.1786 & 0.000477 \\
\hline Mobility 5 & 0.2712 & 0.000479 & 0.2697 & 0.000426 \\
\hline Self-care 2 & 0.0489 & 0.000294 & 0.0476 & 0.000284 \\
\hline Self-care 3 & 0.0829 & 0.000473 & 0.0819 & 0.000475 \\
\hline Self-care 4 & 0.1691 & 0.000493 & 0.1697 & 0.000545 \\
\hline Self-care 5 & 0.2598 & 0.000486 & 0.2589 & 0.000487 \\
\hline Usual activities 2 & 0.0549 & 0.000283 & 0.0553 & 0.000288 \\
\hline Usual activities 3 & 0.0944 & 0.000479 & 0.0952 & 0.000393 \\
\hline Usual activities 4 & 0.1795 & 0.000409 & 0.1798 & 0.000447 \\
\hline Usual activities 5 & 0.2713 & 0.000528 & 0.2758 & 0.000507 \\
\hline Pain/discomfort 2 & 0.0549 & 0.000235 & 0.0531 & 0.000226 \\
\hline Pain/discomfort 3 & 0.0813 & 0.000462 & 0.0808 & 0.000429 \\
\hline Pain/discomfort 4 & 0.2288 & 0.000451 & 0.2283 & 0.000421 \\
\hline Pain/discomfort 5 & 0.4578 & 0.000446 & 0.4579 & 0.000442 \\
\hline $\begin{array}{l}\text { Anxiety/depres- } \\
\text { sion } 2\end{array}$ & 0.0548 & 0.000262 & 0.0551 & 0.000259 \\
\hline $\begin{array}{l}\text { Anxiety/depres- } \\
\text { sion } 3\end{array}$ & 0.0833 & 0.000468 & 0.0824 & 0.000507 \\
\hline $\begin{array}{l}\text { Anxiety/depres- } \\
\text { sion } 4\end{array}$ & 0.1647 & 0.000506 & 0.1611 & 0.000481 \\
\hline $\begin{array}{l}\text { Anxiety/depres- } \\
\text { sion } 5\end{array}$ & 0.3338 & 0.000424 & 0.3337 & 0.000412 \\
\hline$\alpha_{0}$ & -1.6296 & 0.000560 & -1.6435 & 0.000545 \\
\hline$\alpha_{1}$ & 1.0952 & 0.000871 & 1.1088 & 0.000889 \\
\hline $\begin{array}{l}\text { No. of observa- } \\
\text { tions }\end{array}$ & 10,000 & & 9787 & \\
\hline $\begin{array}{l}\text { No. of left-cen- } \\
\text { sored }\end{array}$ & 439 & & 436 & \\
\hline DIC & 9426.56 & & 9121.68 & \\
\hline
\end{tabular}

Variance function for "heteroscedastic censored model with Bayesian estimation" is $\sigma_{j}^{2}=\exp \left(\alpha_{0}+\alpha_{1} \mu_{j}\right)$; MCSE refers to Monte-Carlo Standard Error

\section{Discussion}

The value set reported in this paper will enable evidence on QALYs, based on the preferences of Mexican people, to inform the HTA processes that Mexican healthcare authorities use.

While both cTTO and DCE data were collected, following the EuroQol EQ-5D-5L valuation protocol, the study team decided to base the value set on cTTO data. A number of other countries have also made this decision-including the USA and Uruguay, as noted earlier. Notably, the new EQ-5D-5L value set for the UK, recently announced by
Table 3 Comparison of Mexican, Uruguayan, and USA value-set characteristics

\begin{tabular}{|c|c|c|c|}
\hline & Mexico $^{\mathrm{a}}$ & Uruguay & USA \\
\hline $\begin{array}{l}\text { Proportion of } \\
\text { worse than dead } \\
\text { health states }\end{array}$ & $9.1 \%$ & $0.9 \%$ & $20.0 \%$ \\
\hline Minimum value & -0.596 & -0.264 & -0.573 \\
\hline 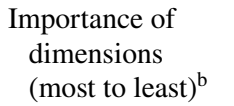 & $\begin{array}{l}\text { PD-AD-UA- } \\
\text { MO-SC }\end{array}$ & MO-SC-PD-UA-AD & $\begin{array}{l}\text { PD-MO- } \\
\text { AD-SC- } \\
\text { UA }\end{array}$ \\
\hline
\end{tabular}

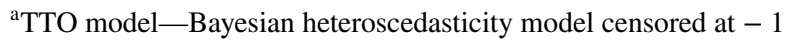

${ }^{\mathrm{b}}$ Based on the relative sizes of the level 5 coefficients

${ }^{\mathrm{c}}$ Pain/discomfort (PD); anxiety/depression (AD); usual activities (UA); mobility (MO); self-care (SC)

NICE and the EuroQol Group, will be based only on cTTO data as well [28]. Given the importance of this work for HTA in Mexico, and the greater acceptance of cTTO as a basis for the value sets to be used in HTA, this was deemed the preferred option a priori. The possibility of a hybrid modelwhere both DCE and cTTO data are modelled together-was considered but rejected, given the lack of consensus regarding hybrid models.

It is important to note the patterns in utility decrements associated with movements between levels on each of the dimensions in the cTTO model. The largest differences in coefficients are evident between levels 5 and 4 (on four of the dimensions), and these differences are particularly marked for pain/discomfort and anxiety/depression. For example, the difference between PD5 and PD4 is 0.2296. These characteristics of the value set will exert an influence on the estimation of QALY gains-for example, suggesting that a one-level improvement from extreme pain/discomfort will yield more QALY gains than a one-level improvement from moderate pain/discomfort, ceteris paribus. The use of the value set to inform cost-effectiveness analysis will reflect these patterns of preferences. The availability of an EQ-5D-5L value set will facilitate local data collection using this instrument to inform future HTA decisions in Mexico. Exactly how the value set is to be used in HTA-for example, whether QALY estimates based on it should be accompanied by sensitivity analyses based on the standard errors-will require consideration and guidance from local decision makers.

Some limitations of the study should be mentioned. The design and methodology followed a well-established international valuation protocol, which involves basing healthstate utility values on the preferences of the general population. This practice is informed by normative arguments (e.g., the argument that preferences ought to be elicited from behind a veil of ignorance rather than from individuals with vested interests) [35]. However, it has been criticized in the 
literature and there have been calls to use preferences from patients (either instead of or in addition to public preferences) [36, 37] as patients are likely to be better informed than the public about what it is like to live in states of impaired health.

Preference data were collected using two techniquescTTO and DCE_-but the value set is based only on TTO data. While there are reasonable grounds for this decision (described above), it means that the DCE data collected will play no role in informing HTA decision making in Mexico. Further, the TTO technique is itself subject to limitations [38] (though it is worth noting that this is true of all stated preference methods). The cTTO and DCE data showed markedly different preference patterns (see Section 2 of the ESM Appendix for an overview of the DCE results). Our existing analyses have not been able to fully explain the reasons for these differences. Qualitative methods-such as think-aloud interviews - may help to explore this issue, but these were not included as part of the current study.

\section{Conclusions}

The EQ-5D-5L value set reported in this study is the first value set produced for any health-related quality of life instrument in Mexico. The provision of a value set, which reflects the distinctive preferences of the adult Mexican general public, will facilitate and support use of EQ-5D-5L in multicenter clinical trials and quality-of-life studies. This will in turn improve the evidence used in HTA to define technologies that might be financed by public healthcare institutions.

Supplementary Information The online version contains supplementary material available at https://doi.org/10.1007/s40258-021-00658-0.

Acknowledgements The authors thank ISPOR Chapter Mexico members for their support during the study and De las Heras-Demotecnia group for the in-field work applying the survey. The authors also thank Elly Stolk, Juan Manuel Ramos-Goñi, Aureliano Finch, and Arnd Jan Prause from the EuroQol Office for their support and valuable comments throughout the study setup and data collection phases.

\section{Declarations}

Funding This study received unrestricted financial support from the Asociación Mexicana de la Industria de Investigación Farmacética (AMIIF) AC and in-kind support from the EuroQol Group for initial training on the EQ-5D-5L valuation protocol of CG, RG, and CC, and access to the EQ-VT tool for the study.

Conflicts of interest ND, KS, and YF are members of the EuroQol Group. CG started the project as member of the Economic Analysis Unit at the Mexican Secretariat of Health. However, she left her position in 2019. RG started the project as a member of General Health
Council. However, she left her position in November 2018. MP and RG are currently employees of the pharmaceutical industry.

Ethics approval The study received ethical approval IMSS/DPM/ UEIPS/CIS/2128/2018 from the National Committee of Ethics and Bioethics and the National Coordination of Research in Health of the Mexican Institute of Social Security and was conducted in accordance with the Declaration of Helsinki.

Consent to participate Informed consent was obtained from all individual respondents included in the study. Respondents were informed about their freedom of refusal. Anonymity and confidentiality were maintained throughout the research process.

Consent for publication Not applicable.

Availability of data and material Not applicable.

Code availability Not applicable.

Authors' contributions The study was conceived by CG, RG, CC, KS, $\mathrm{MP}$, and ND. BZ, YF, and KS performed the descriptive data analysis. $\mathrm{BZ}$ and YF undertook the econometric modelling. CG, KS, ND, BZ, and YF developed the statistical analysis plan. KS and ND reviewed the modelling output. All authors provided their intellectual inputs into the manuscript; CG produced the first draft of the paper, which KS and ND edited. All authors read and approved the final manuscript.

Open Access This article is licensed under a Creative Commons Attribution-NonCommercial 4.0 International License, which permits any non-commercial use, sharing, adaptation, distribution and reproduction in any medium or format, as long as you give appropriate credit to the original author(s) and the source, provide a link to the Creative Commons licence, and indicate if changes were made. The images or other third party material in this article are included in the article's Creative Commons licence, unless indicated otherwise in a credit line to the material. If material is not included in the article's Creative Commons licence and your intended use is not permitted by statutory regulation or exceeds the permitted use, you will need to obtain permission directly from the copyright holder. To view a copy of this licence, visit http://creativecommons.org/licenses/by-nc/4.0/.

\section{References}

1. Consejo Nacional de Población (CONAPO), Proyecciones de la población de México y de las entidades federativas, 2016-2050 y conciliación demográfica de México 1950-2015. https://www. gob.mx/conapo/acciones-y-programas/conciliacion-demograficade-mexico-1950-2015-y-proyecciones-de-la-poblacion-de-mexico-y-de-las-entidades-federativas-2016-2050. Accessed 2 June 2021.

2. Russell Reynolds Associates, The Pharmaceutical Industry in Mexico: New Challenges, New Leaders. https://www.russellrey nolds.com/sites/default/files/mexican_pharm_trendsreport.pdf. Accessed 2 June 2021.

3. Ley General de Salud. Artículo 17, fracción V, artículos 28 y 29. Diario Oficial de la Federación, 7 febrero 2021. https://www. google $. \operatorname{com} / \mathrm{url}$ ? $\mathrm{sa}=\mathrm{t} \& \mathrm{rct}=\mathrm{j} \& \mathrm{q}=\& \mathrm{esrc}=\mathrm{s} \&$ source $=$ web $\& \mathrm{~cd}=\&$ ved=2ahUKEwj68ouCqvnwAhUEWqwKHQW9AC0QFjAAegQ IBBAD\&url=http\%3A\%2F\%2Fwww.diputados.gob.mx\%2FLey esBiblio\%2Fpdf_mov\%2FLey_General_de_Salud.pdf\&usg= AOvVaw1PR3PWmoj8xgUs6BZxVmql. Accessed 2 June 2021. 
4. Consejo de Salubridad General. Reglamento Interior de la Comisión Interinstitucional del Cuadro Básico y Catálogo de Insumos del Sector Salud. Ultima reforma publicada 14 Julio 2017. http://dof.gob.mx/index_111.php?year=2017\&month= $07 \&$ day $=14$. Accessed 2 June 2021.

5. Consejo de Salubridad General. Reglamento Interior del Consejo de Salubridad General, Artículos 9, 15, 17. DOF. 11 diciembre 2009. http://www.csg.gob.mx/descargas/pdf/normatividad/Regla mento_Interior_del_CSG-2013.pdf. Accessed 2 June 2021.

6. Consejo de Salubridad General. Guía para la Conducción de Estudios de Evaluación Económica para la Actualización del Cuadro Básico y Catálogo de Insumos del Sector Salud en México. Comisión Interinstitucional del Cuadro Básico y Catálogo de Insumos del Sector Salud. Diciembre 2017. http://www.csg. gob.mx/descargas/pdf/priorizacion/cuadro-basico/guias/insumos_ salud/GEI_2017_Diciembre.pdf. Accessed 2 June 2021.

7. Consejo de Salubridad General. Guía de Evaluación de Insumos para la Salud. Comisión Interinstitucional del Cuadro Básico y Catálogo de Insumos del Sector Salud. Diciembre 2017. http:// www.csg.gob.mx/descargas/pdf/priorizacion/cuadro-basico/guias/ insumos_salud/GEI_2017_Diciembre.pdf. Accessed 2 June 2021.

8. Richardson J, McKie J, Bariola E. Multiattribute utility instruments and their use. In: Culyer AJ, editor. Encyclopaedia of health economics. San Diego: Elsevier Science; 2014.

9. EuroQol Research Foundation. EQ-5D-5L User Guide, 2019. https://euroqol.org/publications/user-guides. Accessed 2 June 2021.

10. Devlin NJ, Brooks R. EQ-5D and the EuroQol group: past, present and future. Appl Health Econ Health Policy. 2017;15(2):127-37.

11. Rowen D, Zouraq IA, Chevrou-Severac H, van Hout B. International regulations and recommendations for utility data for health technology assessment. Pharmacoeconomics. 2017;35(1):11-9.

12. Kennedy-Martin M, Slaap B, Herdman M, van Reenen M, Kennedy-Martin T, Greiner W, Busschbach J, Boye KS. Which multiattribute utility instruments are recommended for use in cost-utility analysis? A review of national health technology assessment (HTA) guidelines. Eur J Health Econ. 2020;21:1245-57.

13. Rencz F, Gulácsi L, Drummond M, Golicki D, Rupel VP, Simon J, Stolk EA, Brodszky V, Baji P, Závada J, Petrova G. EQ-5D in central and Eastern Europe: 2000-2015. Qual Life Res. 2016;25(11):2693-710.

14. Devlin NJ, Parkin D, Browne J. Patient-reported outcome measures in the NHS: new methods for analysing and reporting EQ-5D data. Health Econ. 2010;19(8):886-905.

15. Ernstsson O, Janssen MF, Heintz E. Collection and use of EQ-5D for follow-up, decision-making, and quality improvement in health care - the case of the Swedish National Quality Registries. J Patient Rep Outcomes. 2020;4:78. https://doi.org/10.1186/ s41687-020-00231-8.

16. Stolk E, Ludwig K, Rand K, van Hout B, Ramos-Goñi JM. Overview, update, and lessons learned from the International EQ-5D-5L valuation work: version 2 of the EQ-5D-5L valuation protocol. Value Health. 2019;22(1):23-30.

17. Instituto Nacional de Estadística y Geografía (INEGI). Regiones Socioeconómicas de México, p. 14-24. http://sc.inegi.gob.mx/ niveles/datosnbi/reg_soc_mexico.pdf. Accessed 2 June 2021 .

18. Asociación Mexicana de Agencias de Investigación de Mercado (AMAI), Niveles socioeconómicos. https://www.amai.org/NSE/ index.php?queVeo=niveles. Accessed 2 June 2021.

19. Oppe M, Rand-Hendriksen K, Shah K, Ramos-Goñi JM, Luo N. EuroQol protocols for time trade-off valuation of health outcomes. Pharmacoeconomics. 2016;34(10):993-1004.
20. Oppe, M. and van Hout, B. 2017. The "power" of eliciting EQ5D-5L values: the experimental design of the EQ-VT. EuroQol Working Paper. Rotterdam: EuroQol.

21. Augustovski F, Rey-Ares L, Irazola V, Garay OU, Gianneo O, Fernández G, Morales M, Gibbons L, Ramos-Goñi JM. An EQ5D-5L value set based on Uruguayan population preferences. Qual Life Res. 2016;25(2):323-33.

22. Versteegh MM, Vermeulen KM, Evers SM, de Wit GA, Prenger R, Stolk EA. Dutch tariff for the five-level version of EQ-5D. Value Health. 2016;19(4):343-435.

23. Xie F, Pullenayegum E, Gaebel K, Bansback N, Bryan S, Ohinmaa A, Poissant L, Johnson JA. A time trade-off-derived value set of the EQ-5D-5L for Canada. Med Care. 2016;54(1):98-105.

24. Rencz F, Brodszky V, Gulácsi L, Golicki D, Ruzsa G, Pickard AS, Law EH, Péntek M. Parallel valuation of the EQ-5D-3L and EQ-5D-5L by time trade-off in Hungary. Value Health. 2020;23(9):1235-45.

25. Pickard AS, Law EH, Jiang R, Pullenayegum E, Shaw JW, Xie F, Oppe M, Boye KS, Chapman RH, Gong CL, Balch A. United States valuation of EQ-5D-5L health states using an international protocol. Value Health. 2019;22(8):931-41.

26. Shah KK, Ramos-Goñi JM, Kreimeier S, Devlin NJ. An exploration of methods for obtaining $0=$ dead anchors for latent scale EQ5D-Y values. Eur J Health Econ. 2020. https://doi.org/10.1007/ s10198-020-01205-9.

27. Ramos-Goñi JM, Pinto-Prades JL, Oppe M, Cabasés JM, Serrano-Aguilar P, Rivero-Arias O. Valuation and modeling of EQ-5D-5L health states using a hybrid approach. Med Care. 2017;55(7):e51-8.

28. EuroQol, 2020. New UK EQ-5D-5L Valuation Study. https:// euroqol.org/eq-5d-instruments/eq-5d-5l-about/valuation-stand ard-value-sets/new-uk-eq-5d-51-valuation-study_blog/. Accessed 2 June 2021

29. Ramos-Goñi JM, Oppe M, Slaap B, Busschbach JJ, Stolk E. Quality control process for EQ-5D-5L valuation studies. Value Health. 2017;20(3):466-73.

30. Engel L, Bansback N, Bryan S, Doyle-Waters MM, Whitehurst DG. Exclusion criteria in national health state valuation studies: a systematic review. Med Decis Mak. 2016;36(7):798-810.

31. Ludwig K, von der Schulenburg JMG, Greiner W. German value set for the EQ-5D-5L. Pharmacoeconomics. 2018;36(6):663-74.

32. Devlin N, Shah K, Feng Y, Mulhern B, van Hout B1. Valuing health-related quality of life: an EQ-5D-5L value set for England. Health Econ. 2018;27:7-22.

33. StataCorp. Stata: Release 16. Statistical Software. College Station: StataCorp LLC; 2019. p. 112-254.

34. Augustovski F, Belizán M, Gibbons L, Reyes N, Stolk E, Craig BM, Tejada RA. Peruvian valuation of the EQ-5D-5L: a direct comparison of time trade-off and discrete choice experiments. Value Health. 2020;23(7):880-8.

35. Gold MR, Siegel JE, Russell LB, Weinstein MC. Cost-effectiveness in health and medicine. New York: Oxford University Press; 1996.

36. Versteegh MM, Brouwer WBF. Patient and general public preferences for health states: a call to reconsider current guidelines. Soc Sci Med. 2016;165:66-74.

37. Slejko JF, Mattingly TJ II, Mullins CD, Perfetto EM. Future of patients in healthcare evaluation: the patient-informed reference case. Value Health. 2019;22(5):545-8.

38. Lugnér AK, Krabbe PF. An overview of the time trade-off method: concept, foundation, and the evaluation of distorting factors in putting a value on health. Expert Rev Pharmacoecon Outcomes Res. 2020;20(4):331-42. 


\section{Authors and Affiliations}

\section{Cristina Gutierrez-Delgado ${ }^{1,2} \cdot$ Rosa-María Galindo-Suárez ${ }^{3,4} \cdot$ Cesar Cruz-Santiago $^{3,4} \cdot$ Koonal Shah $^{5}$. Manny Papadimitropoulos ${ }^{6,7} \cdot$ Yan Feng $^{8} \cdot$ Bernarda Zamora $^{9} \cdot$ Nancy Devlin ${ }^{9,10}$}

Rosa-María Galindo-Suárez

rosamaria.galindo.suarez@gmail.com

Cesar Cruz-Santiago

cealcruz@gmail.com

Koonal Shah

KoonalShah@phmr.com

Manny Papadimitropoulos

papadimitropoulos_manny@lilly.com

Yan Feng

yan.feng@qmul.ac.uk

Bernarda Zamora

bernardazamora@gmail.com

Nancy Devlin

nancy.devlin@unimelb.edu.au

1 Faculty of Sciences, National Autonomous University of Mexico, Mexico City, Mexico
2 Economic Analysis Unit, Secretariat of Health, Mexico City, Mexico

3 General Health Council, Mexico City, Mexico

4 ISPOR Chapter Mexico, Mexico City, Mexico

5 PHMR Ltd, London, UK

6 Eli Lilly Canada Inc, Toronto, Canada

7 University of Toronto, Toronto, Canada

8 Institute of Population Health Sciences, Queen Mary University of London, London, UK

9 Office of Health Economics, London, UK

10 Centre for Health Policy, University of Melbourne, Melbourne, VIC, Australia 